\title{
Detection and characterization of a novel copper-dependent intermediate in a lytic polysaccharide monooxygenase
}

\author{
Raushan K. Singh ${ }^{1}$, Bart v. Oort ${ }^{4}$, Benedikt Möllers ${ }^{2}$, David A. Russo ${ }^{3}$, Ranjitha Singh ${ }^{1}$, \\ Høgni Weihe ${ }^{1}$, Manish K. Tiwari ${ }^{1}$, Roberta Croce ${ }^{4}$, Paul E. Jensen ${ }^{3}$, Claus Felby ${ }^{2}$, Morten J. \\ Bjerrum $^{1 *}$ \\ ${ }^{1}$ Department of Chemistry, University of Copenhagen, Copenhagen, Denmark \\ ${ }^{2}$ Department of Geosciences and Natural Resource Management, University of Copenhagen, \\ Frederiksberg C, Denmark \\ ${ }^{3}$ Department of Plant and Environmental Sciences, University of Copenhagen, Frederiksberg \\ C, Denmark
}

${ }^{4}$ Biophysics of Photosynthesis, Vrije Universiteit Amsterdam, Amsterdam, Netherlands

*Correspondence author. Email: mobj@chem.ku.dk 


\section{Abstract}

Lytic polysaccharide monooxygenases (LPMOs) are copper-containing enzymes capable of oxidizing crystalline cellulose and the enzyme has large practical application in the process of refining biomass. The LPMO catalytic mechanism still remains debated despite several proposed reaction mechanisms. Here, we report a long-lived intermediate ( $t_{1 / 2}=6-8$ minutes $)$ observed in an LPMO from Thermoascus aurantiacus (TaLPMO9A). The intermediate with a strong absorption around $420 \mathrm{~nm}$ is formed when reduced $\mathrm{LPMO}-\mathrm{Cu}(\mathrm{I})$ reacts with $\mathrm{H}_{2} \mathrm{O}_{2}$. UVvis absorption spectroscopy, electron paramagnetic resonance (EPR), and stopped-flow spectroscopy indicate that the observed long-lived intermediate involves the copper center and a nearby tyrosine (Tyr175). We propose that the reaction with $\mathrm{H}_{2} \mathrm{O}_{2}$ first forms a highly reactive short-lived $\mathrm{Cu}$ (III)-intermediate which is subsequently transformed into the observed longlived copper-dependent intermediate. Since sub-equimolar amount of $\mathrm{H}_{2} \mathrm{O}_{2}$ to LPMO boosts oxidation of phosphoric acid swollen cellulose (PASC) suggests that the long-lived copperdependent intermediate is part of the catalytic mechanism for LPMOs. The proposed mechanism offers new perspectives in the oxidative reaction mechanism of copper enzymes and hence for the biomass oxidation and the reactivity of copper in biological systems. 


\section{INTRODUCTION}

The cleavage of carbon-hydrogen bonds is central to the biological breakdown of organic matter into carbon dioxide and water. With the discovery of lytic polysaccharide monooxygenases (LPMOs), it was shown for the first time, how an enzyme with a single copper in the active site can activate dioxygen to break carbon-hydrogen bonds and degrade the most abundant and recalcitrant polysaccharides, such as cellulose and chitin ${ }^{1}$. Since their discovery, LPMOs have been found in fungi, bacteria, virus, invertebrates, and algae, highlighting their biological importance ${ }^{2}$. The diversity of identified LPMOs has increased with currently six different families classified in the CAZY database as auxiliary activity (AA) AA9, AA10, AA11, AA13, AA14 and AA15 families ${ }^{3,4}$. However, despite the importance of LPMOs and the extensive research in their structure and enzymology, the actual catalytic mechanism is yet to be resolved.

LPMOs have a solvent-exposed mononuclear type-2 copper active site, with a t-shaped coordination sphere named the histidine-brace, where two histidine residues provide three nitrogen ligands, two from $\mathrm{N}-\mathrm{His}$ and one from the terminal amine ${ }^{5}$. Another characteristic of LPMOs is that the copper also coordinates to an adjacent aromatic amino acid; for AA9, AA11, AA13, AA14, and AA15 LPMOs the amino acid is a tyrosine, whereas for AA10 LPMOs it is mostly a phenylalanine with few exceptions ${ }^{6}$. The overall reaction of LPMOs has been proposed to be the abstraction of a hydrogen atom from the glycoside $\mathrm{C}-\mathrm{H}$ bond followed by insertion of an oxygen atom in the carbohydrate chain and thereby cleaving the glycosidic bond 7. Specifically, for glucans, the oxidation of the pyranose ring can take place at either the $\mathrm{C} 1$ position producing aldonic acids or at the $\mathrm{C} 4$ position producing 4-ketoaldose (gemdiols) ${ }^{8}$. The catalytic cycle is believed to be initiated by the reduction of LPMO-Cu(II) to LPMO-Cu(I), by an external electron donor, followed by binding of dioxygen to LPMO-Cu(I) ${ }^{9}$. The nature 
of the external electron donor varies widely and four LPMO electron donor systems have been reported: fungal metabolites, lignin/phenols, GMC oxidoreductases and photoexcited pigments 10-15. The function of LPMOs has been subject to great controversy ${ }^{15-18}$. In the currently established model LPMOs are seen as monooxygenases. However, this view is challenged by new data indicating that both $\mathrm{O}_{2}$ and $\mathrm{H}_{2} \mathrm{O}_{2}$ can function as co-substrates ${ }^{15,17,18}$. This is further complicated by the fact that LPMOs can generate $\mathrm{H}_{2} \mathrm{O}_{2}$ when the enzyme is not bound to the substrate, a pathway referred to as the "futile cycle" ${ }^{15}$. A copper-bound oxygen intermediate has been shown by neutron scattering ${ }^{7,19,20}$, but the nature of the oxygen intermediate remains unknown. Kjaergaard et al. (2014) using DFT modelling suggested the presence of a $\left[\mathrm{CuO}_{2}\right]^{+}$ superoxide species. Other theoretical works have shown that the superoxide in $\left[\mathrm{CuO}_{2}\right]^{+}$is not sufficiently reactive to abstract a hydrogen from the glycoside $\mathrm{C}-\mathrm{H}$ bond ${ }^{19,21}$ and have suggested that copper-oxyl, $[\mathrm{CuO}]^{+}$copper-hydroxy, $[\mathrm{CuOH}]^{2+}$ or copper-hydroperoxide $[\mathrm{CuOOH}]$ species ${ }^{7,22-26}$ may be generated by reductions and protonations of e.g. the superoxide in $\left[\mathrm{CuO}_{2}\right]^{+}$. Walton and Davis analyzed a wide range of possible catalytic cycles with different oxygen intermediates and divided them into two classes based on the LPMO being free or bound to a substrate ${ }^{22,27}$. They also indicated that some of the suggested pathways could involve $\mathrm{Cu}(\mathrm{III})$ as a reactive species.

The possible formation of $\mathrm{Cu}(\mathrm{III})$ in mono-nuclear copper sites is supported by earlier studies. The oxidation of copper complexes with tri- and tetrapeptides has been reported by Margerum and co-workers ${ }^{28-30}$, showing that it was possible to form $\mathrm{Cu}(\mathrm{III})$ complexes with a lifetime of several minutes. $\mathrm{Cu}$ (III) complexes generated by mixing the copper-coordinated tetrapeptide $\left(\mathrm{Cu}\right.$ (II)Gly2HisGly) with ascorbate, and $\mathrm{H}_{2} \mathrm{O}_{2}$ decomposed to relatively stable alkene peptides ${ }^{30}$. The oxidation could also be performed electrochemically or by strong oxidants (e.g. $\left.\left[\mathrm{Ir}(\mathrm{IV}) \mathrm{Cl}_{6}\right]^{2-}\right)^{30}$. The formed copper(III)-tetrapeptide showed UV-vis absorption around $400 \mathrm{~nm}$ with a molar absorbance of $1.7 \cdot 10^{3} \mathrm{M}^{-1} \mathrm{~cm}^{-1}{ }^{30}$. Comparing the ligand 
environment of the $\mathrm{Cu}(\mathrm{II})$ site in the tetrapeptides with that of the $\mathrm{Cu}(\mathrm{II})$ site in LPMOs shows that the $\mathrm{Cu}(\mathrm{II})$ sites in LPMOs have large structural similarity with these simple $\mathrm{Cu}(\mathrm{II})$ peptide complexes. A major difference between the structure of LPMOs ${ }^{5}$ and the copper(II)tetrapeptide complexes is that the $\mathrm{Cu}$ (II) in LPMOs is interacting with the nearby aromatic amino acid (tyrosine (Tyr175) in TaLPMO9A). Interestingly, tyrosine radical is reported to have molar absorbance of $2.5 \cdot 10^{3}-5.0 \cdot 10^{3}$ at $420 \mathrm{~nm}^{31}$. It is also worth mentioning that ascorbate has been used as redox cofactor and $\mathrm{H}_{2} \mathrm{O}_{2}$ as co-substrate for LPMO-catalyzed oxidation $^{5,15,32}$.

It was therefore investigated if a possible $\mathrm{Cu}(\mathrm{III})$ and/or tyrosine radical species were formed when LPMOs was treated with various combinations of ascorbate and $\mathrm{H}_{2} \mathrm{O}_{2}$. A new LPMO intermediate with absorption around $420 \mathrm{~nm}$ involving the active site copper and a nearby tyrosine was observed and its reactivity, formation and decay were studied using spectroscopic and chromatographic tools. 


\section{RESULTS}

\section{Oxidation of TaLPMO9A-Cu(II) by ascorbate and $\mathrm{H}_{2} \mathrm{O}_{2}$}

To test if copper in LPMOs could be oxidized to $\mathrm{Cu}(\mathrm{III})$ as seen for copper coordinated to triand tetrapeptides ${ }^{30}$, we oxidized the LPMO by ascorbate and $\mathrm{H}_{2} \mathrm{O}_{2}$. For this study, we chose a well-characterized AA9 LPMO from Thermoascus aurantiacus (TaLPMO9A) also known as TaGH61A/TaAA9A ${ }^{5}$. Highly pure and fully $\mathrm{Cu}$ (II) loaded holoTaLPMO9A (Cu/LPMO molar ratio is $1.07 \pm 0.06$ ) was obtained as outlined in Materials and Methods (Fig. S1). Impurities from the protein including media impurities were removed and the enzyme was highly pure as judged from SDS-PAGE (see Fig. S1B). UV-vis spectra (Fig. 1) shows the changes occurring when purified holoTaLPMO9A is mixed with ascorbate followed by the addition of subequimolar amounts of $\mathrm{H}_{2} \mathrm{O}_{2}$ (7:1 protein: $\mathrm{H}_{2} \mathrm{O}_{2}$ ratio). The UV-vis spectra shows an absorption peak appearing at $420 \mathrm{~nm}$ after addition of ascorbate and $\mathrm{H}_{2} \mathrm{O}_{2}$ (Fig. 1A); this peak decreases with time, having nearly disappeared after $45 \mathrm{~min}$. (Fig. 1A and1B). In this experiment, the $420 \mathrm{~nm}$ peak decay is close to a first-order decay with a half-life of approximately 6 - 8 minutes (Fig. 1B). As shown in Fig. S2, a decrease in $\mathrm{Cu}(\mathrm{II})$ absorbance around $640 \mathrm{~nm}$ and in the CD signal around $720 \mathrm{~nm}$ after addition of ascorbate confirmed that the LPMO-Cu(II) was partially reduced to $\mathrm{LPMO}-\mathrm{Cu}(\mathrm{I})$ before the addition of $\mathrm{H}_{2} \mathrm{O}_{2}$. The $420 \mathrm{~nm}$ peak is not observed when the apoLPMO is treated with ascorbate and $\mathrm{H}_{2} \mathrm{O}_{2}$ (Fig. 2). Furthermore, no peak is observed at $420 \mathrm{~nm}$ when holoTaLPMO9A is oxidized with $\mathrm{H}_{2} \mathrm{O}_{2}$ in the absence of ascorbate or when $\mathrm{H}_{2} \mathrm{O}_{2}$ is added to holoTaLPMO9A before addition of ascorbate. This suggests that the presence of copper is essential and that LPMO-Cu(II) cannot form the product absorbing at $420 \mathrm{~nm}$ by reaction with $\mathrm{H}_{2} \mathrm{O}_{2}$ alone, and that the reduction of $\mathrm{LPMO}-\mathrm{Cu}(\mathrm{II})$ to $\mathrm{LPMO}-\mathrm{Cu}(\mathrm{I})$ is essential for the formation of the $420 \mathrm{~nm}$ peak. Margerum and co-workers have also reported the lack of oxidation of $\mathrm{Cu}$ (II)-( $\left.\mathrm{H}_{-2} \mathrm{Gly}_{2} \mathrm{HisGly}\right)^{-}$to $\mathrm{Cu}$ (III) with $\mathrm{H}_{2} \mathrm{O}_{2}$ alone ${ }^{30}$. 

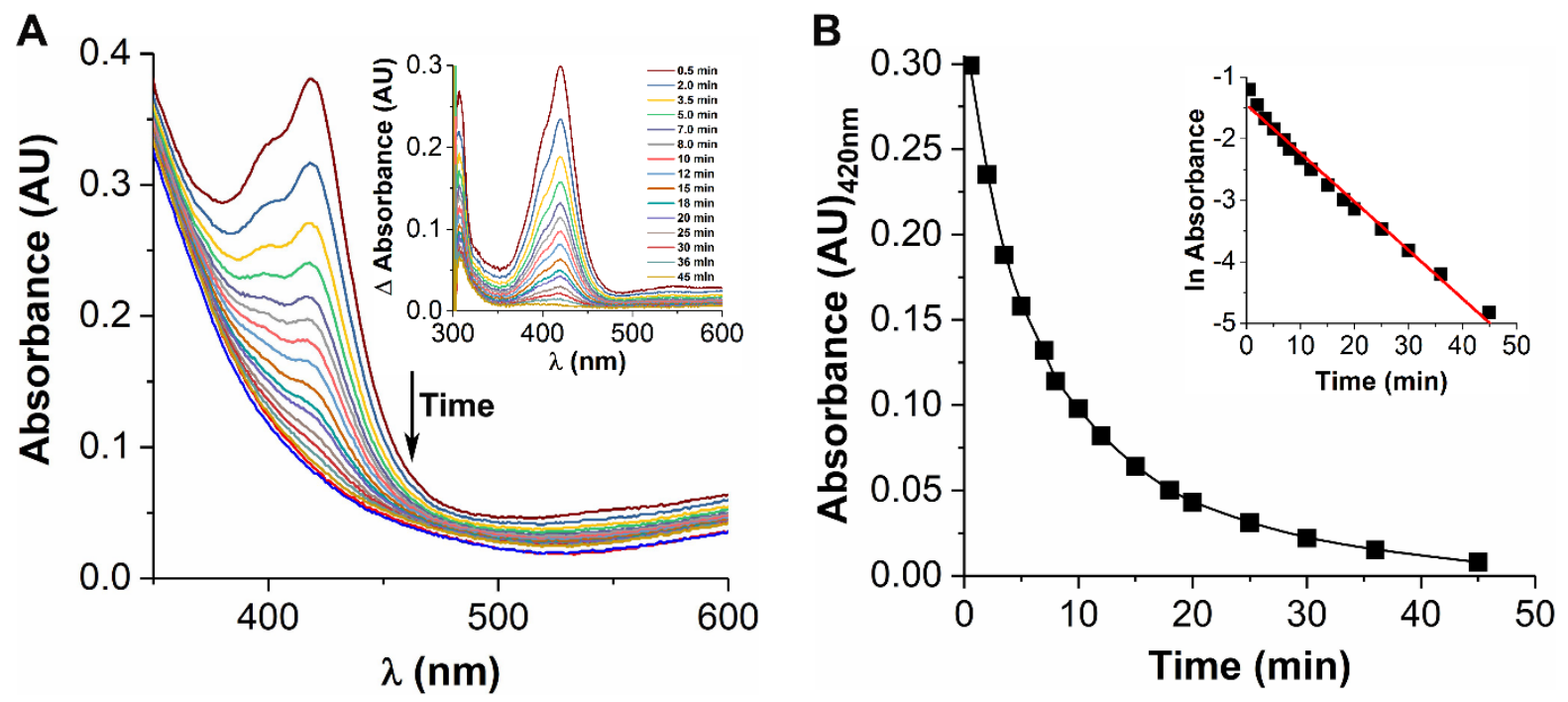

Fig. 1. UV-vis spectral change when TaLPMO9A is mixed with ascorbate and $\mathrm{H}_{2} \mathrm{O}_{2}$. (A)

UV-vis spectra of reduced TaLPMO9A after addition of sub-equimolar concentrations of $\mathrm{H}_{2} \mathrm{O}_{2}$. Red and blue lines represent the holoTaLPMO9A and reduced holoTaLPMO9A spectra, respectively. After addition of $\mathrm{H}_{2} \mathrm{O}_{2}$ to reduced holoTaLPMO9A peak at $420 \mathrm{~nm}$ appears which decays with time. The inset shows the peak at $420 \mathrm{~nm}$ at a different time intervals after subtracting the reduced holoTaLPMO9A spectra from reduced holoTaLPMO9A $+\mathrm{H}_{2} \mathrm{O}_{2}$ spectra. (B) Decay of the absorption peak at $420 \mathrm{~nm}$ over time. The inset shows the linear plot of ln absorbance over time. Experimental conditions: TaLPMO9A $1.39 \mathrm{mM}$, ascorbate $2 \mathrm{mM}$, $\mathrm{H}_{2} \mathrm{O}_{2} 0.2 \mathrm{mM}$ in $20 \mathrm{mM}$ phosphate buffer at $\mathrm{pH} 6.6$ and room temperature.

\section{Formation and decay kinetics of intermediate}

The formation and decay of the intermediate were investigated by stopped-flow kinetics. By addition of $0.1 \mathrm{mM} \mathrm{H}_{2} \mathrm{O}_{2}\left(2: 1\right.$ protein: $\mathrm{H}_{2} \mathrm{O}_{2}$ ratio) the intermediate is formed in less than $10 \mathrm{~s}$ and then gradually decays with a lifetime of around $6-8$ minutes (Fig. 2A and 2B). For apoTaLPMO9A (i.e. copper free), no intermediate is observed (Fig. 2A and 2B). Subsequent stopped-flow experiments at varying concentrations of $\mathrm{H}_{2} \mathrm{O}_{2}$ (Fig. 2C) show that increasing amounts of $\mathrm{H}_{2} \mathrm{O}_{2}$ reduces the formation and lifetime of the intermediate. A simple bi- 
exponential fit to the data indicates that the decay reaction contains both a first- and a secondorder component (Fig. 2D, Table S1). This suggests that the decay proceeds both as a "secondorder" reaction with $\mathrm{H}_{2} \mathrm{O}_{2}$ ( $k_{1}$ increases with $\mathrm{H}_{2} \mathrm{O}_{2}$ concentration) and as a "first-order" decay $\left(k_{2}\right)$. Furthermore, $\mathrm{pH}$ variation (5.9 to 7.6 ) had only small effect on the formation and decay of the intermediate (Fig. S3).
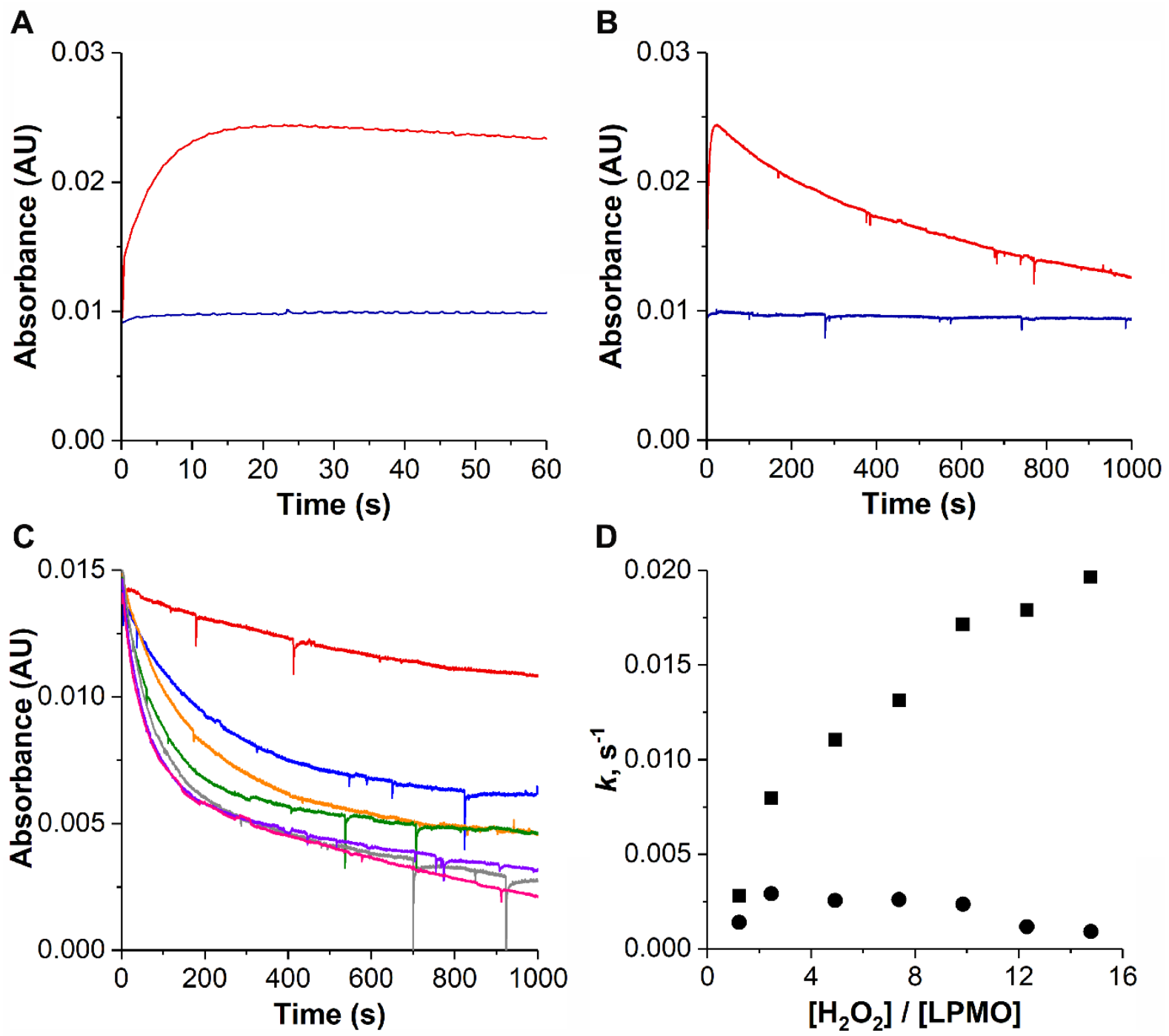

Fig. 2. Single wavelength absorption transients at $420 \mathrm{~nm}$ for the reaction of reduced TaLPMO9A with $\mathrm{H}_{2} \mathrm{O}_{2}$. Transients absorbance observed for $60 \mathrm{~s}(\mathbf{A})$ and $1000 \mathrm{~s}$ after the addition of $\mathrm{H}_{2} \mathrm{O}_{2}(\mathbf{B})$. Red and blue lines represent the holoTaLPMO9A and apoTaLPMO9A, respectively. Experimental conditions: apoTaLPMO9A or holoTaLPMO9A $0.2 \mathrm{mM}$, ascorbate $1 \mathrm{mM}, \mathrm{H}_{2} \mathrm{O}_{2} 0.1 \mathrm{mM}$ in $20 \mathrm{mM}$ phosphate buffer at pH 6.6 and $20{ }^{\circ} \mathrm{C}$. (C) Transients 
absorbance during the reaction of reduced TaLPMO9A with varying concentrations of $\mathrm{H}_{2} \mathrm{O}_{2}$ observed for $1000 \mathrm{~s}$. Red, blue, orange, green, violet, grey and pink lines represent $0.25,0.5$, 1.0, 1.5, 2.0, 2.5 and $3.0 \mathrm{mM} \mathrm{H}_{2} \mathrm{O}_{2}$, respectively. Rate constants were determined by fitting the transients to equation 2 (Materials and methods) and are given in the supplementary information. Fitting was performed for the time period 5 to $1000 \mathrm{~s}$. Absorbance was normalized to same peak intensity and plotted against time (D). Fitted rate constants as a function of varying $\mathrm{H}_{2} \mathrm{O}_{2}$ concentrations for reduced TaLPMO9A. Rates, $k_{1}$ (fast) and $k_{2}$ (slow) represented as solid square and solid circle, respectively. Only data collected after $5 \mathrm{~s}$ were analyzed and fitted. Experimental conditions: holoTaLPMO9A $0.2 \mathrm{mM}$, ascorbate $1 \mathrm{mM}$ in 20 $\mathrm{mM}$ phosphate buffer at $\mathrm{pH} 6.6$ and $20^{\circ} \mathrm{C}$.

\section{Paramagnetic behaviour of intermediate}

The intermediate, formed at sub-equimolar amounts of $\mathrm{H}_{2} \mathrm{O}_{2}$ had a lifetime of around 6 - 8 minutes. It is therefore possible to investigate the nature of the intermediate using EPR spectroscopy. The EPR spectrum of the holoTaLPMO9A/ascorbate/ $\mathrm{H}_{2} \mathrm{O}_{2}$ system was obtained at $77 \mathrm{~K}$ (Fig. S4). The EPR spectra of partly reduced TaLPMO9A and partly reduced TaLPMO9A with addition of 0.5 equivalents $\mathrm{H}_{2} \mathrm{O}_{2}$ were essentially identical to those of TaLPMO9A (Fig. S4). The parameters for the EPR spectra at $77 \mathrm{~K}$ are given in Table S2. The observed EPR spectrum is characteristic of a type 2 copper site with $g_{z}(g \|)=2.27$ and $g_{x y}(g \perp)$ $=2.06$ and $A_{\mathrm{z}}(A \|)=492 \mathrm{MHz}$. Furthermore, addition of ascorbate reduced the signal without changing the obtained parameters (Table S2).

Next, to mimic the conditions in which we observed the intermediate at $420 \mathrm{~nm}$ in the UV-vis experiment, the EPR spectra were measured at room temperature. The spectrum of holoTaLPMO9A in solution, in the presence of both ascorbate and ascorbate $/ \mathrm{H}_{2} \mathrm{O}_{2}$ is shown in Fig. 3A and the fit parameters are given in Table 1 . The parameters $g_{z}=2.27$ and $g_{x y}=2.06$ and 
$A_{\mathrm{z}}=459 / \mathrm{MHz}$ are close to those obtained at $77 \mathrm{~K}$. Furthermore, in correlation with the reduction of TaLPMO9A-Cu(II) to TaLPMO9A-Cu(I) addition of ascorbate reduced the signal to about half intensity without changing the obtained parameters as seen from $\mathrm{Cu}$ (conc.) in Table 1. The EPR spectrum after addition of both ascorbate and $\mathrm{H}_{2} \mathrm{O}_{2}$ has an increased signal with some broadening. The difference in EPR signal in Fig. 3B is obtained by subtracting the reduced TaLPMO9A spectra (blue) from the spectrum of the ascorbate $/ \mathrm{H}_{2} \mathrm{O}_{2}$ treated TaLPMO9A (green). The parameters for this difference spectrum are $g_{z}=2.17$ and $g_{x y}=2.07$.

A

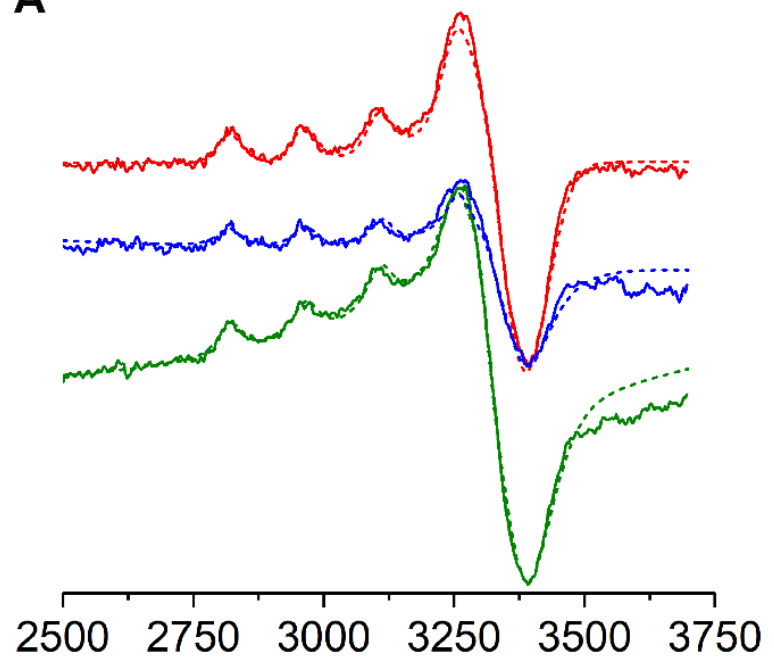

Field / Gauss
B

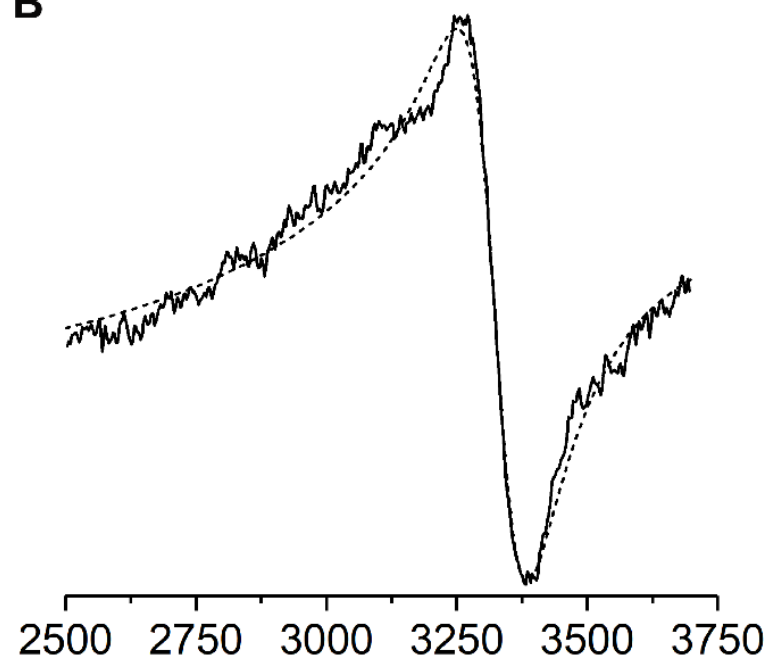

Field / Gauss

Fig. 3. EPR spectra of holoTaLPMO9A. (A) X-band EPR spectra of TaLPMO9A (red), reduced TaLPMO9A (blue) and $\mathrm{H}_{2} \mathrm{O}_{2}$ treated reduced TaLPMO9A (green) at room temperature in $20 \mathrm{mM}$ phosphate buffer, $\mathrm{pH}$ 6.6. The sample was put in a capillary and then carefully placed inside the quartz EPR tube. The spectrum recorded for the buffer in the capillary was used as the reference to remove the background signal. (B) Spectra after subtracting the TaLPMO9A spectra from $\mathrm{H}_{2} \mathrm{O}_{2}$ treated reduced TaLPMO9A spectra. Solid lines indicate experimental spectra recorded of solutions at room temperature. The dashed lines indicate computed spectra obtained with parameters reported in Table 1. Experimental conditions: $3.6 \mathrm{mM}$ TaLPMO9A, $4 \mathrm{mM}$ ascorbate, and $2 \mathrm{mM} \mathrm{H}_{2} \mathrm{O}_{2}$ at $\mathrm{pH} 6.6$ in $20 \mathrm{mM}$ phosphate buffer. 
Table 1. EPR spectral parameters for TaLPMO9A at room temperature. I, and II denote TaLPMO9A, and reduced TaLPMO9A, respectively. IV denotes the EPR parameters of the spectra after subtracting the reduced TaLPMO9A spectra from $\mathrm{H}_{2} \mathrm{O}_{2}$ treated reduced TaLPMO9A spectra.

\begin{tabular}{cccc}
\hline Parameters & I & II & IV \\
\hline $\mathrm{Cu}\left(\mathrm{A}_{\mathrm{z}}\right)(\mathrm{MHz})$ & $459(1)$ & $459(2)$ & - \\
$\mathrm{Cu}\left(\mathrm{g}_{\mathrm{xy}}\right)$ & $2.0637(1)$ & $2.0638(2)$ & - \\
$\mathrm{Cu}\left(\mathrm{g}_{\mathrm{z}}\right)$ & $2.2670(3)$ & $2.2658(4)$ & - \\
$\mathrm{Cu}($ conc. $)$ & $852(17)$ & $409(15)$ & - \\
\hline $\mathrm{S}=1\left(\mathrm{~g}_{\mathrm{xy}}\right)$ & - & - & $2.067(2)$ \\
$\mathrm{S}=1\left(\mathrm{~g}_{\mathrm{z}}\right)$ & - & - & $2.17(1)$ \\
$\mathrm{S}=1$ (conc. $)$ & - & - & $636(37)$ \\
\hline
\end{tabular}

\section{Influence of the intermediate formation on LPMO activity}

To elucidate the potential role of the observed intermediate in the catalytic cycle of the LPMO, we used a substrate conversion assay with PASC as substrate. The released oligomeric products were detected and quantified by high-performance anion exchange chromatography (HPAEC) (Fig. 4). At $0.1 \mathrm{mM} \mathrm{H}_{2} \mathrm{O}_{2}$, the release of non-oxidized oligosaccharides derived from PASC oxidation was enhanced whereas the release of C4-oxidized products only showed minor differences relative to a sample without addition of exogenous $\mathrm{H}_{2} \mathrm{O}_{2}$ (Fig. 4A). The correlation to the mechanism when only $\mathrm{O}_{2}$ is present is further elaborated in the discussion. As previously observed from Stopped-flow experiments, the lifetime of the intermediate is approximately inversely correlated with the concentration of $\mathrm{H}_{2} \mathrm{O}_{2}$. We therefore, proceeded with experiments in the presence of 20 times higher $\mathrm{H}_{2} \mathrm{O}_{2}$ concentration (Fig. 4B). Interestingly, only a negligible release of $\mathrm{C} 4$ oxidation products was detected which is in accordance with the level of observed intermediate. 

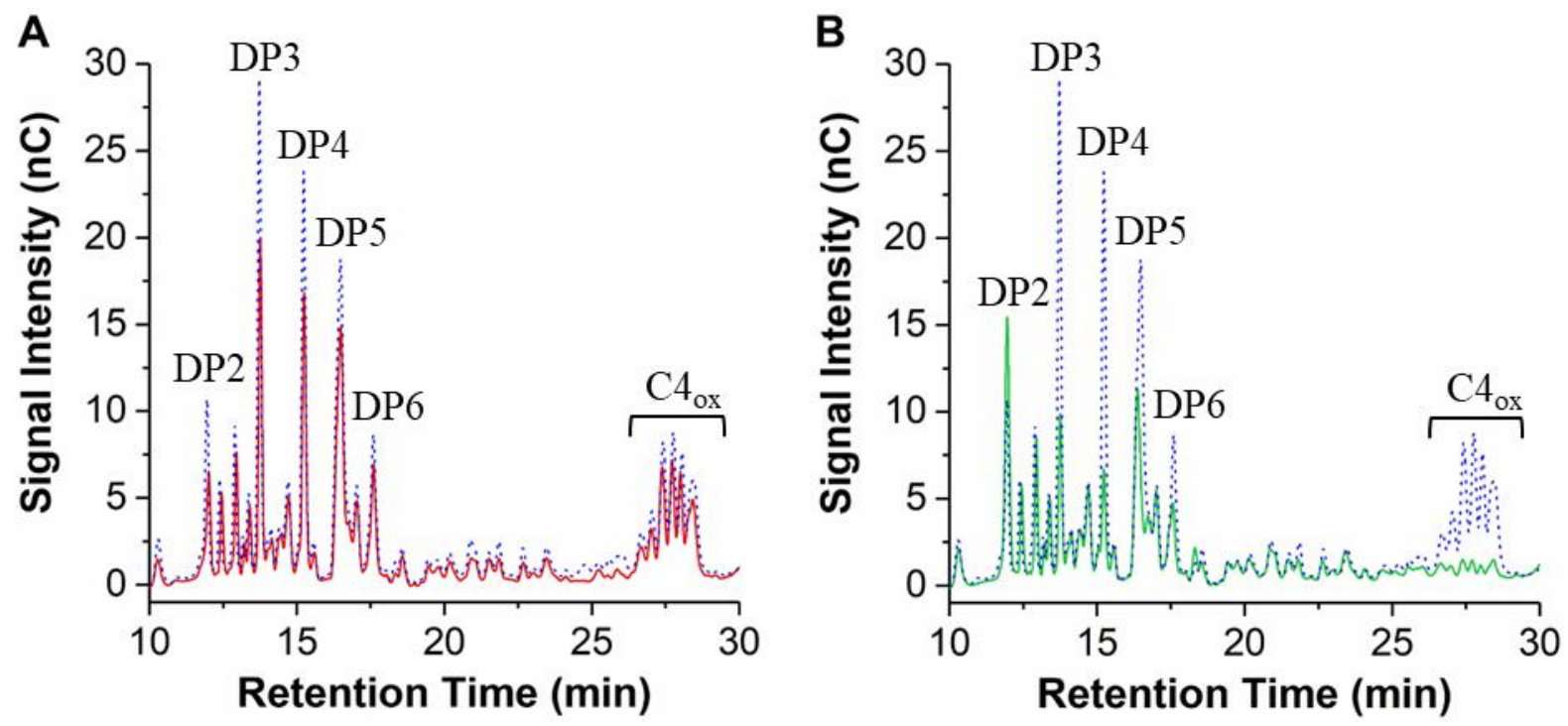

Fig. 4. TaLPMO9A catalyzed oxidation of PASC. (A) HPAEC chromatogram showing the TaLPMO9A catalyzed oxidation of PASC with supplementation of $0.1 \mathrm{mM} \mathrm{H}_{2} \mathrm{O}_{2}$ (blue) and without $\mathrm{H}_{2} \mathrm{O}_{2}$ (red). (B) TaLPMO9A catalyzed oxidation of PASC was supplemented with 0.1 $\mathrm{mM} \mathrm{H} \mathrm{O}_{2}$ (blue) and $2.0 \mathrm{mM} \mathrm{H} \mathrm{O}_{2}$ (green). Experimental conditions: TaLPMO9A (0.1 mM), PASC (0.4\%), ascorbate $(2 \mathrm{mM})$, in phosphate buffer $(20 \mathrm{mM}, \mathrm{pH} 6.0)$ at $25{ }^{\circ} \mathrm{C}$ and $800 \mathrm{rpm}$ for $30 \mathrm{~min}$. The peaks were assigned according to the elution profile of commercially available non-oxidized cello-oligosaccharides. DP2, cellobiose; DP3, cellotriose; DP4, cellotetraose; DP5, cellopentaose; DP6, cellohexaose. The C4-oxidized glucose (4-keto aldose) peaks appear after a retention time of $25 \mathrm{~min}$.

\section{LPMO oxidation and dimerization}

Finally, the post-assay samples were analyzed by SDS-PAGE, which revealed dimer formation of the TaLPMO9A (Fig. 5A). The dimerization can only be observed with the complete holoTaLPMO9A/ascorbate/ $\mathrm{H}_{2} \mathrm{O}_{2}$ system and no dimers were formed in reaction mixtures where only LPMO and ascorbate were present, or in the absence of $\mathrm{Cu}$ (apoLPMO) (Fig. 5A, Fig. S5A, Fig. S5B and Fig. 6B). The LPMO used in this study has 12 tyrosine residues and their positions are shown in the X-ray crystal structure in Fig. S5C ${ }^{5}$. It is conceivable that the 
observed dimerization could be due to intermolecular dityrosine formation. To confirm this hypothesis, we followed the fluorescence at $405 \mathrm{~nm}$, the emission wavelength expected for dityrosine ${ }^{33}$, over a period of 24 hours (Fig. 5B). The observed increase in fluorescence over time strongly indicates that the dimer formation is, in fact, due to dityrosine cross-linking between LPMOs in solution. Interestingly, this dimer formation is only seen under conditions where the intermediate is observed and occurs over a period of 24 hours which is much slower than the decay of the intermediate (Fig. 1B). Based on the observation from SDS-PAGE that the formation of the dimers is between 10 to $15 \%$ of the whole LPMO sample it is estimated that the molar absorbance of the LPMO intermediate at $420 \mathrm{~nm}$ is between 1400 to $2200 \mathrm{M}^{-}$ ${ }^{1} \mathrm{~cm}^{-1}$ (using data from Fig. 1A and 5A), assuming that two intermediate will result in one dimer. Which is within the range of other reported values on similar systems ${ }^{31}$.

To probe if dimer formation had an effect on enzymatic activity, the LPMO dimers were purified by size exclusion chromatography (Fig. 6A and B). The two separate peaks observed by size exclusion chromatography and the two bands observed on SDS-PAGE clearly indicate that the oxidized TaLPMO9A exhibits intermolecular cross-linkage. Furthermore, the purified monomeric and dimeric fractions were tested for activity with an amplex red assay. The dimers maintained $\sim 90 \%$ of the monomer activity (Fig. 6C). Furthermore, the peroxidase activity of TaLPMO9A was checked using 2,6-dimethoxyphenol (2,6-DMP) assay ${ }^{34}$. The 2,6DMP assay with TaLPMO9A showed that $\mathrm{H}_{2} \mathrm{O}_{2}$ could activate TaLPMO9A (Fig. S6). As shown in Fig. S6, the recorded spectra show the appearance of a peak right after the initiation of the reaction showing maxima at $469 \mathrm{~nm}$ (final reaction product coerulignone). This peak at $469 \mathrm{~nm}$ was less prominent (80-fold less $\mathrm{OD}_{469 \mathrm{~nm}}$ ), when the reaction was performed in the absence of $\mathrm{H}_{2} \mathrm{O}_{2}$ (Fig. S6B and S6C). We also checked the peroxidase activity of oxidized TaLPMO9A (Fig. S8). Similarly, the peroxidase activity of oxidized TaLPMO9A (for 
TaLPMO9A oxidation, the reduced TaLPMO9A was treated with 0.5 and 10 eq of $\mathrm{H}_{2} \mathrm{O}_{2}$, and then purified before checking the peroxidase activity) retained $91 \%$ and $78 \%$ of its activity.

A

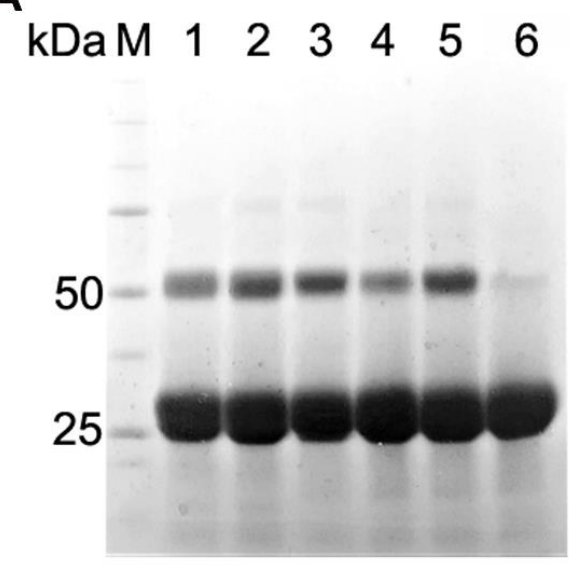

B

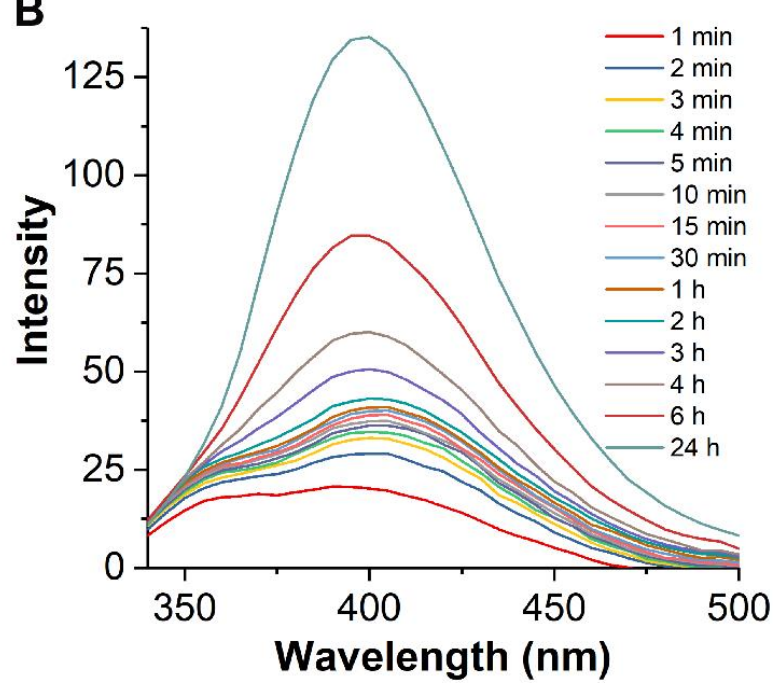

Fig. 5. The appearance of TaLPMO9A dimers correlates with the formation of dityrosine.

(A) SDS-PAGE analysis of the TaLPMO9A after initial intermediate formation. Lane 1: TaLPMO9A $+2 \mathrm{mM}$ ascorbate $+2 \mathrm{mM} \mathrm{H}_{2} \mathrm{O}_{2}$, Lane 2: TaLPMO9A $+1 \mathrm{mM}$ ascorbate $+1 \mathrm{mM}$ $\mathrm{H}_{2} \mathrm{O}_{2}$, Lane 3: TaLPMO9A $+1 \mathrm{mM}$ ascorbate $+0.5 \mathrm{mM} \mathrm{H}_{2} \mathrm{O}_{2}$, Lane 4: TaLPMO9A $+0.5 \mathrm{mM}$ ascorbate $+0.2 \mathrm{mM} \mathrm{H}_{2} \mathrm{O}_{2}$, Lane 5: TaLPMO9A $+1 \mathrm{mM}$ ascorbate $+0.2 \mathrm{mM} \mathrm{H}_{2} \mathrm{O}_{2}$, Lane 6: TaLPMO9A (1.39 mM). (B) Dityrosine formation followed by fluorescence spectroscopy with emission at $340-500 \mathrm{~nm}$ and excitation at $320 \mathrm{~nm}$. The difference in fluorescence spectra is obtained by subtracting the reduced TaLPMO9A spectra from $\mathrm{H}_{2} \mathrm{O}_{2}$ treated reduced TaLPMO9A spectra. Assay was performed on Jasco FP-6300 spectrofluorometer. Experimental conditions: TaLPMO9A $(1.05 \mathrm{mM})+$ ascorbate $(2 \mathrm{mM})+\mathrm{H}_{2} \mathrm{O}_{2}(0.5 \mathrm{mM})$ incubated in phosphate buffer (20mM, pH 6.6) at room temperature. 

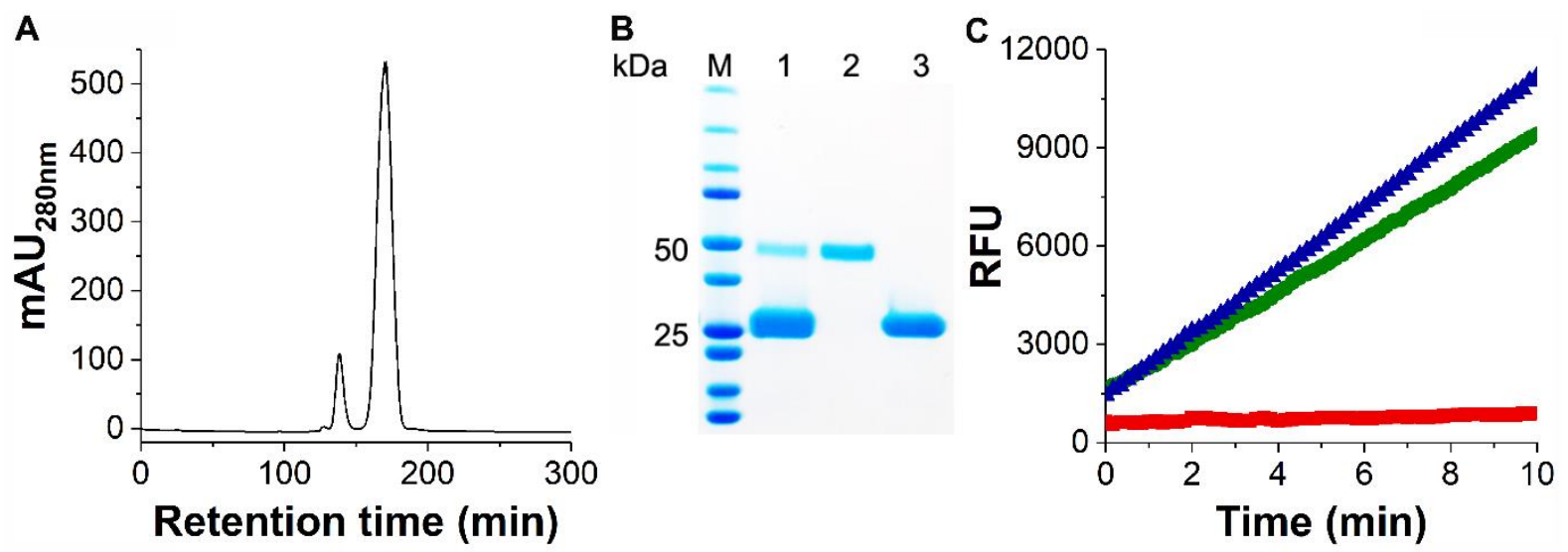

Fig. 6. Purification of observed dityrosine dimers of TaLPMO9A and the activity as measured by Amplex red assay. (A) Chromatogram showing purification of oxidized TaLPMO9A using Hiload 26/60 Superdex 75 prep grade column. Oxidized TaLPMO9A was washed and concentrated before loading into the size exclusion column. Monomer and dimer peaks were well separated and eluted separately. (B) SDS-PAGE analysis of the purified TaLPMO9A after oxidation. Lane 1, oxidized TaLPMO9A, lane 2, purified dimer LPMO, lane 3, purified monomer LPMO. (C) Amplex red assay was performed using purified dimer and monomer LPMO. Green $(\bullet)$ and blue $(\boldsymbol{\Delta})$ symbols represent activity for purified dimer and purified monomer LPMO, respectively. Red ( $\square$ ) symbols represent reaction mixture without LPMO. The sample composition was: Ascorbate $(0.08 \mathrm{mM})$, EDTA $(0.1 \mathrm{mM})$, Amplex red (0.05 mM), HRP (20 U), purified-TaLPMO9A $(2.0 \mu \mathrm{g} / \mathrm{mL})$. The assay was performed on SpectraMax M2 multi-detection microplate reader using excitation and emission at 557 and $583 \mathrm{~nm}$. 


\section{DISCUSSION}

The observed long-lived intermediate seen in the UV-Vis spectra of TaLPMO9A at $420 \mathrm{~nm}$ (Fig. 1), is formed by treating TaLPMO9A solution with ascorbate and $\mathrm{H}_{2} \mathrm{O}_{2}$. The presence of copper at the active site of TaLPMO9A is essential for the formation of the intermediate since no intermediate is formed when apoTaLPMO9A is used in the experiment (Fig. 2A and B). Treating holoTaLPMO9A with $1 \mathrm{mM}$ ascorbate solution results in partial reduction of LPMO$\mathrm{Cu}$ (II) to LPMO-Cu(I) as seen from the UV-vis and EPR measurements of TaLPMO9A with and without ascorbate (Fig. S2 and Fig. 3). Furthermore, the stopped-flow experiments (Fig. 2) showed that approximately 0.5 equivalents of $\mathrm{H}_{2} \mathrm{O}_{2}$ seem to give the highest observed level of the intermediate when using $1 \mathrm{mM}$ ascorbate. Additionally, the holoTaLPMO9A upon mixing with $\mathrm{H}_{2} \mathrm{O}_{2}$ in the absence of ascorbate, does not show any intermediate formation. Combining the two observations, this is a strong indication that $\mathrm{H}_{2} \mathrm{O}_{2}$ only reacts with TaLPMO9A-Cu(I) during the formation of the intermediate. It is, therefore, possible that TaLPMO9A-Cu(I) with $\mathrm{H}_{2} \mathrm{O}_{2}$ forms LPMO-Cu(III). This conclusion is supported by Margerum and coworkers observation of $\mathrm{Cu}(\mathrm{III})$ formation when they treated tri- and tetrapeptides of $\mathrm{Cu}$ (II) with strong oxidants or ascorbate/ $\mathrm{H}_{2} \mathrm{O}_{2}{ }^{28-30}$. It is important to note that the absorption around $420 \mathrm{~nm}$ from the LPMO intermediate disappears completely with the decay of the intermediate. Indicating that we do not see absorbance from stable oxidation products of TaLPMO9A like alkenes as described by Margerum and co-workers ${ }^{30}$. However, we do observe the formation of dityrosine. This indicates that the decay mechanism of a $\mathrm{Cu}$ (III) species is different in the LPMOs compared to what happens when $\mathrm{Cu}(\mathrm{III})$ is formed in simple tri- and tetrapeptides. The active site in LPMO is somehow protected from oxidation by the possibly formed $\mathrm{Cu}(\mathrm{III})$.

The intermediate forms within seconds after mixing $\mathrm{H}_{2} \mathrm{O}_{2}$ with reduced TaLPMO9A as seen from the stopped-flow experiments (Fig. 2A). When no substrate and no surplus $\mathrm{H}_{2} \mathrm{O}_{2}$ is 
present, the intermediate is quite stable with a half-life of 6 - 8 min (Fig. 1). However, stoppedflow experiments with increasing amount of $\mathrm{H}_{2} \mathrm{O}_{2}$ clearly showed that the intermediate not only forms faster but decays even faster (Fig. 2C). The bi-exponential fit to the stopped-flow data indicated that the decay reaction contains both a first-order component as well as a secondorder component proportional to the added $\mathrm{H}_{2} \mathrm{O}_{2}$ (Fig. 2D, Table S1) even if these data are not collected under strict pseudo-first order conditions. The average value $\left(2.0 \cdot 10^{-3} \mathrm{~s}^{-1}\left(k_{2}\right)\right)$ for the first-order reaction obtained from stopped-flow experiment fits well with the value of $1.7 \cdot 10^{-3}$ $\mathrm{s}^{-1}$ obtained from data in Fig. 1. Furthermore, the second-order decay is dominant with surplus $\mathrm{H}_{2} \mathrm{O}_{2}$ which explains why this intermediate has been so difficult to observe.

The chemical nature of the observed intermediate was investigated by X-band EPR spectroscopy (Fig. S4). It is clear that the $77 \mathrm{~K}$ EPR spectrum of TaLPMO9A is as expected when comparing with spectra already published in the literature ${ }^{5,35}$. EPR measurements were also performed at room temperature to mimic conditions as close as possible to the stoppedflow experiments. Addition of ascorbate reduces the intensity of the EPR signal similar to what is expected when approximately half of the $\mathrm{Cu}(\mathrm{II})$ in the enzyme is reduced to $\mathrm{Cu}(\mathrm{I})$. Addition of 0.5 equivalents $\mathrm{H}_{2} \mathrm{O}_{2}$ to the partly reduced TaLPMO9A showed an increase in the EPR signal, and some line broadening (Fig. 3A). This could be indicative of a compound with an $S$ $=1$ signal growing up underneath the $\mathrm{Cu}(\mathrm{II})$ signal ${ }^{31}$. Fitting of composite spectra with overlapping signals resulted, as expected, in poorly determined and highly correlated parameters. To quantify the concentration of the $S=1$ species better, we subtracted the blue trace from the green trace resulting in the broad signal shown in Fig. 3B. Subtracting the two spectra gave an EPR signal (Fig. 3B) that was fitted with $g_{z}=2.17$ and $g_{x y}=2.07$. This EPR signal is probably due to a ferromagnetically coupled triplet state arising in the TaLPMO9A from exchange coupling of two unpaired spins. Notice that the average value, 2.10(2), of the $g$ factors for this species, is lower than that, 2.1311(4), of the $\mathrm{Cu}^{2+}$ signal. This is in agreement 
with the interpretation of this as originating from an $S=1$ multiplet being a result of an interaction of $\mathrm{Cu}^{2+}$ with an organic radical. Canters and co-workers studied a radical intermediate in a small laccase (SLAC) formed in the presence of oxygen and ascorbate giving rise to an absorption band at $410 \mathrm{~nm}^{31}$. The spin system was identified as a tyrosine radical ferromagnetically coupled to a $\mathrm{Cu}$ (II) giving rise to a triplet state. The active tyrosine was identified from the crystal structure of the SLAC as a Tyr108 radical coupled to the spin half of the T2 Cu in SLAC positioned approximately $5 \AA$ away ${ }^{31,36,37}$. Based on the similarity with the intermediate observed in SLAC, we propose that the observed intermediate of TaLPMO9A is due to the $\mathrm{Cu}$-ion in the active site of the protein interacting with Tyr175 positioned approximately $2.79 \AA$ away from it (Fig. S7) ${ }^{5,22,38}$. Given the shorter distance compared to SLAC, it is conceivable to have favourable interaction between the active site copper and Tyr175. This suggests that the observed long-lived intermediate is actually a CuTyrintermediate. A possible explanation for the formation of this CuTyr-intermediate could be that LPMO-Cu(I) is reacting with $\mathrm{H}_{2} \mathrm{O}_{2}$ forming a short-lived LPMO-Cu(III) (and $2 \mathrm{HO}^{-}$). This LPMO-Cu(III) would likely coordinate with the proximate Tyr175 and form a $\mathrm{Cu}(\mathrm{III})$ combined with tyrosyl deprotonation. Such a structure is isoelectronic with a $\mathrm{Cu}(\mathrm{II})$ interaction with a tyrosyl radical and this resonance will stabilize the system and could explain the broad and weak EPR signal observed underneath the $\mathrm{Cu}(\mathrm{II})$ signal. An EPR signal is only expected from the $\mathrm{Cu}(\mathrm{II})-{ }^{-} \mathrm{OTyr}$ structure since $\mathrm{Cu}(\mathrm{III})$ and a deprotonated tyrosyl in $\mathrm{Cu}(\mathrm{III})-{ }^{-} \mathrm{OTyr}$ do not have unpaired electrons. The reason why an EPR signal is not seen at $77 \mathrm{~K}$ could be that the equilibrium is shifted towards the $\mathrm{Cu}(\mathrm{III})$ - $^{-} \mathrm{OTyr}$ structure and/or that the interaction changes in favor of an anti ferromagnetically coupling to $\mathrm{Cu}(\mathrm{II})$ at low temperature.

The kinetic data in Fig. 2C shows that the process for the disappearance of the CuTyrintermediate can occur via two different pathways. The observed apparent first-order process is slow with a first-order half-life of 6 - 8 minutes. Treatment of reduced TaLPMO9A with 
sub-equimolar $\mathrm{H}_{2} \mathrm{O}_{2}$ reduce the enzyme activity slightly since the amplex red assay and 2,6DMP assay showed more than 90\% activity (Fig. 6 and Fig. S8A). The observed apparent second order process causing a decay of the intermediate in TaLPMO9A is dependent on the concentration of $\mathrm{H}_{2} \mathrm{O}_{2}$. This process seems to be comparatively more detrimental than the first order process since the activity of the TaLPMO9A after treatment with 10 eq of $\mathrm{H}_{2} \mathrm{O}_{2}$ reduced to $78 \%$ (see Fig. S8B). The decay pathway of the CuTyr-intermediate is somehow correlated with the formation of dityrosines since dityrosin formation only is observed when the CuTyrintermediate is formed. The dityrosine could be formed due to intra- or inter-molecular covalent linkage resulting in a TaLPMO9A dimer ${ }^{8,39,40}$. Our observations indicates that $\mathrm{H}_{2} \mathrm{O}_{2}$ can indeed be a substrate for the LPMOs but only at low concentrations. As soon as $\mathrm{H}_{2} \mathrm{O}_{2}$ concentration increases from 0.5 to 10 eq (20-times), it is detrimental to the enzymatic oxidation of cellulose (Fig. 4) ${ }^{15}$. This is also supported by studies on the gene expressions showing that expression of LPMO is closely followed by the expression of catalase ${ }^{41}$. This indicates that in nature $\mathrm{H}_{2} \mathrm{O}_{2}$ is controlled to be at low concentration to avoid unwanted oxidative processes inactivating the LPMOs.

Based on experimental studies, we have observed that a CuTyr-intermediate is formed when $\mathrm{LPMO}-\mathrm{Cu}(\mathrm{I})$ and $\mathrm{H}_{2} \mathrm{O}_{2}$ react at room temperature. We propose that the observed CuTyrintermediate is a real intermediate in the catalytic cycle of AA9 LPMO and therefore a key to understanding the catalytic mechanism of AA9 LPMOs which have a tyrosine in the active site (Fig. 7). The first product from this reaction between LPMO-Cu(I) and $\mathrm{H}_{2} \mathrm{O}_{2}$ is probably a short-lived $\mathrm{Cu}$ (III) intermediate as described by Margerum and co-workers ${ }^{30}$. In case of no substrate, this reactive $\mathrm{Cu}(\mathrm{III})$ intermediate is transformed into the more stable CuTyrintermediate to protect the active site from oxidation, for example in the form of alkene formation as seen in tetrapetides ${ }^{30}$. In presence of substrate, it is possible that the $\mathrm{Cu}(\mathrm{III})$ intermediate can react directly with the substrate thus not forming the CuTyr-intermediate. It 
is yet to be established if this CuTyr-intermediate is reactive enough to abstract a hydrogen atom from a C-H bond. However, our observation on PASC oxidation supplemented with $\mathrm{H}_{2} \mathrm{O}_{2}$ (Fig. 4A) and of the dityrosine formation (Fig. 5) indicate that this CuTyr-intermediate acts as a powerful oxidant. We believe that the proposed CuTyr-intermediate is also important for the classical superoxo mechanism ${ }^{7,19}$. In that mechanism, $\mathrm{LPMO}-\mathrm{Cu}(\mathrm{I})$ binding to $\mathrm{O}_{2}$ is followed by a rapid reoxidation in an inner-sphere mechanism forming $\mathrm{LPMO}-\mathrm{Cu}(\mathrm{II})-\left(\mathrm{O}_{2}{ }^{-{ }^{-}}\right)^{9,19,23,42}$. Calculations have shown that this $\mathrm{LPMO}-\mathrm{Cu}(\mathrm{II})-\left(\mathrm{O}_{2}{ }^{-}\right)$intermediate is not able to abstract a hydrogen atom from a C-H bond by itself ${ }^{7,25}$. However, studies by Kjaergaard et al. (2014) have indicated that the bound superoxide radical can be replaced by water thus releasing $\mathrm{HO}_{2} /$ $\mathrm{O}_{2}{ }^{\cdot-}$ into solution which can further be transformed into $\mathrm{H}_{2} \mathrm{O}_{2}{ }^{19}$. It is known from several studies that LPMO indeed produces $\mathrm{H}_{2} \mathrm{O}_{2}$ in case no substrate is present ${ }^{43,44}$. A possible mechanism for the reaction of reduced LPMOs with $\mathrm{O}_{2}$ as primary substrate (see Fig. 7 upper right) is thus a release of $\mathrm{O}_{2}^{-}$which disproportionates to $\mathrm{H}_{2} \mathrm{O}_{2}$ in solution. The formed $\mathrm{H}_{2} \mathrm{O}_{2}$ is then used by reduced LPMO to produce the active CuTyr-intermediate. Our stopped-flow studies indicate that LPMO-Cu(I) react very fast with $\mathrm{H}_{2} \mathrm{O}_{2}$.

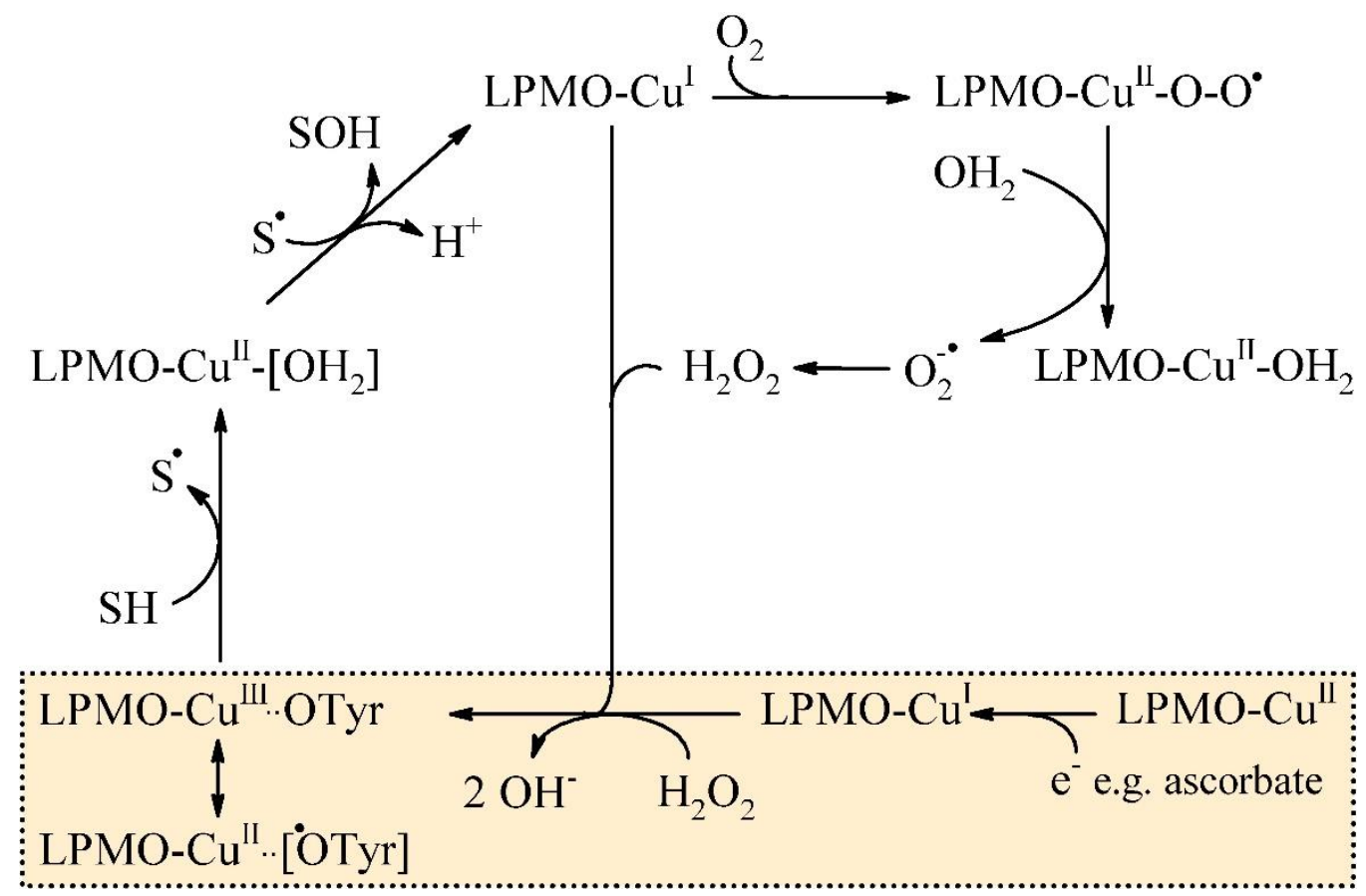


Fig. 7. Proposed mechanism for LPMO catalyzed oxidation of polysaccharides. One electron reduction of the LPMO-Cu(II) to LPMO-Cu(I) occurs, which then reacts with $\mathrm{H}_{2} \mathrm{O}_{2}$ forming the CuTyr-intermediate indicated in the box. The right-side of the upper path, which is reported to produce $\mathrm{H}_{2} \mathrm{O}_{2}$ from the replacement of the superoxide radical ${ }^{9,19,23,26,42}$ also supports the formation of CuTyr-intermediate. Then the resulting CuTyr-Intermediate could abstract a hydrogen atom from the substrate leading to hydroxylation of the substrate. The regenerated LPMO-Cu(I) can then enter a new catalytic cycle. 


\section{CONCLUSION}

In this paper, we demonstrate that $\mathrm{Cu}(\mathrm{I})$ at the active site of TaLPMO9A directly interacts with $\mathrm{H}_{2} \mathrm{O}_{2}$ and results in the formation of a CuTyr-intermediate. TaLPMO9A takes part in a process similar to the $\mathrm{Cu}$ (III) formation found by treating tri- and tetrapeptides of $\mathrm{Cu}$ (II) with ascorbate/ $\mathrm{H}_{2} \mathrm{O}_{2}{ }^{28,30}$. However, in the case of TaLPMO9A the absorbance at $420 \mathrm{~nm}$ slowly disappears without any alkene formation but with dityrosine formation. This indicates that the active site in TaLPMO9A is protected by an additional mechanism not found in simple tri- and tetrapeptides. The reaction scheme is probably first a formation of a $\mathrm{Cu}(\mathrm{III})$ intermediate with a very short lifetime $(<1 \mathrm{~s})$. This could be the active species in LPMO. In the absence of substrate, this $\mathrm{Cu}(\mathrm{III})$ species is transformed into a more stable intermediate in order to protect the active site. EPR data suggest that this long-lived intermediate could be a ferromagnetically coupled $\mathrm{Cu}(\mathrm{II}){ }^{-}$Tyr175 pair. However, it is still not clear if the CuTyr-intermediate is able to oxidize the substrate by itself. Our data indicate that it can react with tyrosines at the surface of TaLPMO9A resulting in the formation of dityrosine bonds. Importantly, the formation and decay of the CuTyr-intermediate do not affect the TaLPMO9A activity significantly as judged from the amplex red assay and the 2,6-DMP assay. 


\section{METHODS}

\section{Materials}

All chemicals were of the highest purity grade commercially available and were purchased from Sigma-Aldrich unless stated otherwise. Ampliflu Red (10-acetyl-3,7dihydroxyphenoxazine) was purchased from Cayman chemical company. $\mathrm{H}_{2} \mathrm{O}_{2}(30 \%$, stabilizer free) purchased from Merck Chemicals GmbH (Darmstadt, Germany). Recombinant TaLPMO9A from T. auranticus expressed in Aspergillus oryzae was donated by Novozymes A/S (Denmark).

\section{Purification and Copper Reconstitution}

Crude TaLPMO9A from Novozymes was filtered using $0.22 \mu \mathrm{M}$ filter and injected into a ÄKTA chromatography system equipped with Hiload 26/60 Superdex 75 prep grade column (Pharmacia Biotech) equilibrated with $20 \mathrm{mM}$ MOPS buffer (pH 7.0). TaLPMO9A was eluted in single peak using MOPS buffer with the flow rate of $1 \mathrm{mLmin}^{-1}$. The fractions corresponding to the single peak were pooled and concentrated using an Amicon Ultra -15 centrifugal filter (3 kDa, Merck Millipore Ltd. Ireland). Copper-(I) chloride was added to the purified TaLPMO9A in a $5 \mathrm{~mL}$ tube on ice under anaerobic environment. After copper reconstitution, the LPMO was injected again into the ÄKTA (equipped with Hiload 26/60 Superdex 75 prep grade column) to obtain fully-loaded purified TaLPMO9A. TaLPMO9A was then concentrated and buffer exchanged to $20 \mathrm{mM}$ phosphate buffer ( $\mathrm{pH}$ 6.6) with an Amicon Ultra -15 centrifugal filter. The purity of TaLPMO9A was analyzed on sodium dodecyl sulfatepolyacrylamide gel electrophoresis (SDS-PAGE) using a Mini-PROTEAN TGX precast gel from Bio-Rad Laboratories with a gradient of 4-15\%. Protein bands were visualized by staining with Coomassie blue and unstained Precision Plus Protein Standard was used for mass determination. 
apoTaLPMO9A was prepared by dialyzing the purified TaLPMO9A extensively using D-tube Dialyzer Maxi (6-8 kDa) from Millipore (Billerica, MA, USA). Dialysis was performed in $20 \mathrm{mM}$ phosphate buffer ( $\mathrm{pH}$ 7) supplemented with $10 \mathrm{mM}$ ethylenediaminetetraacetic acid (EDTA) for $12 \mathrm{hrs}$ at $4{ }^{\circ} \mathrm{C}$. Purified TaLPMO9A was loaded into a D-tube and placed in a beaker containing $600 \mathrm{~mL}$ ice-cold $20 \mathrm{mM}$ phosphate buffer $(\mathrm{pH}$ 7) supplemented with $10 \mathrm{mM}$ EDTA. Buffer was mixed continuously using a magnetic stirrer. During the course of dialysis, buffer was changed twice. After dialysis, the TaLPMO9A was concentrated, the buffer exchanged, and the $\mathrm{Cu}$ concentration was determined using inductively coupled plasma mass spectroscopy (ICP-MS).

\section{Copper and Protein Concentration}

Protein concentration of purified TaLPMO9A was determined by its absorbance at $280 \mathrm{~nm}$ using a NanoDrop spectrophotometer from Fisher Scientific. Extinction coefficient of TaLPMO9A was calculated from TaLPMO9A sequence using online tool available at https://web.expasy.org/protparam/. Copper concentrations were determined using high resolution inductively coupled plasma mass spectroscopy (ICP-MS) on an Aurora M90 ICPMS system from Bruker. For this, TaLPMO9A was diluted in 1.5\% nitric acid. Each sample was measured in triplicate and average values are reported.

\section{LPMO activity assay}

Horseradish peroxidase (HRP)-coupled Amplex red assay was performed on SpectraMax M2 multi-detection microplate reader using black 96-well plate with clear bottom from Nunc ${ }^{44}$. All the reactions were performed in $20 \mathrm{mM}$ sodium phosphate buffer, $\mathrm{pH} 6.0$ at $37{ }^{\circ} \mathrm{C} .200 \mu \mathrm{L}$ of reaction mixture contained $100 \mu \mathrm{M}$ EDTA, $80 \mu \mathrm{M}$ ascorbate, $50 \mu \mathrm{M}$ ampliflu red, $20 \mathrm{UmL}^{-}$ ${ }^{1} \mathrm{HRP}$ and, $2.0 \mu \mathrm{gmL}^{-1}$ LPMO. Reaction was initiated by adding TaLPMO9A and resorufin fluorescence was recorded with an excitation and emission wavelength of $557 \mathrm{~nm}$ and $583 \mathrm{~nm}$, respectively. Fluorescence was measured for $10 \mathrm{~min}$ with $5 \mathrm{sec}$ shaking before the 
measurement. A linear relation between fluorescence and $\mathrm{H}_{2} \mathrm{O}_{2}$ concentrations in the range of $0.1-2 \mu \mathrm{M} \mathrm{H}_{2} \mathrm{O}_{2}$ was observed and the slope $\left(28450\right.$ counts $\left.\mu \mathrm{mol}^{-1}\right)$ was used for the calculation of an enzyme factor to convert the fluorimeters readout (counts $\mathrm{min}^{-1}$ ), into enzyme activity. LPMO activity was defined as one $\mu \mathrm{mol} \mathrm{H}_{2} \mathrm{O}_{2}$ generated per minute under the defined assay conditions.

LPMO activity assay using 2,6-dimethoxyphenol (2,6-DMP) was performed as described by Breslmayr et al. (2018) ${ }^{34}$. The standard reaction mixture for the LPMO activity assay contained $100 \mu \mathrm{M} \mathrm{H}_{2} \mathrm{O}_{2}, 1.0 \mathrm{mM}$ 2,6-DMP and $4 \mu \mathrm{M}$ of TaLPMO9A. Reaction was performed in phosphate buffer $(\mathrm{pH} 7.5)$ at room temperature. Reaction was initiated by adding TaLPMO9A and UV-vis spectra was recorded every 30 seconds for $10 \mathrm{~min}$ on Agilent UVvisible ChemStation.

\section{LPMO oxidation}

LPMO oxidation was achieved by mixing the LPMO with equimolar amounts of L-ascorbate and various concentrations of $\mathrm{H}_{2} \mathrm{O}_{2}{ }^{30}$. LPMO was prepared in $20 \mathrm{mM}$ phosphate buffer ( $\mathrm{pH}$ 6.6) while ascorbate and $\mathrm{H}_{2} \mathrm{O}_{2}$ stock solutions were prepared in deionized filtered $(0.22 \mu \mathrm{m})$ Milli-Q water. The concentration of $\mathrm{H}_{2} \mathrm{O}_{2}$ was confirmed by UV spectroscopy based on the absorption at $265 \mathrm{~nm}\left(\mathcal{E} 10 \mathrm{M}^{-1} \mathrm{~cm}^{-1}\right){ }^{45}$. UV-Vis spectral change was recorded using a SHIMADZU UV-3600 UV-VIS-NIR spectrophotometer. Spectra were recorded from 300 to $700 \mathrm{~nm}$ wavelength. All the solutions were freshly prepared and stored in dark tubes on ice prior to the experiment.

\section{Detection of dityrosine}

TaLPMO9A was oxidized by mixing equimolar concentrations of L-ascorbate and $\mathrm{H}_{2} \mathrm{O}_{2}$. Change in the intrinsic fluorescence of TaLPMO9A due to the formation of dityrosine was recorded at 340-500 nm after excitation at $320 \mathrm{~nm}^{33}$, on a Jasco FP-6300 spectrofluoromater using a $1 \mathrm{~cm}$ cuvette. Spectral changes were monitored at different time points for 24 hours. 


\section{EPR Spectroscopy}

EPR spectra were recorded for reduced, oxidized and unmodified apoTaLPMO9A and holoTaLPMO9A on a Bruker Elexsys E500 equipped with a Bruker ER 4116 DM dual mode cavity spectrometer equipped with a SuperX CW-EPR bridge, and a nitrogen flow cryostat (Helitran; Advance Research Systems). EPR spectra were recorded at room temperature as well as $77 \mathrm{~K}$ using microwave frequency, $9.63 \mathrm{GHz}$; microwave power, 10 milliwatts; modulation amplitude, $8 \mathrm{G}$; and attenuation $10 \mathrm{~dB}$. For room temperature measurements, samples were loaded in capillary tube and then placed into EPR quartz tubes. Theoretical EPR spectra were fitted to the experimental ones as detailed in ref ${ }^{46}$. To reproduce the $\mathrm{Cu}^{2+}$ signals we used the spin Hamiltonian

$\hat{H}=A_{z} \hat{S}_{z} \hat{I}_{z}+\mu_{\mathrm{B}}\left(g_{x} \hat{S}_{x} B_{x}+g_{y} \hat{S}_{y} B_{y}+g_{z} \hat{S}_{z} B_{z}\right)$

Eq. 1

with $\mathrm{S}=1 / 2$ and $\mathrm{I}=3 / 2$ pertinent to the electronic spin doublet and the $\mathrm{Cu}$ nucleus, respectively. Initially, we included in the spin Hamiltonian the $x$ and $y$ components of the hyperfine interaction. However, the experimental data did not warrant such a detailed analysis. Therefore, equation (1) was used in the fitting of all $\mathrm{Cu}^{2+}$ signals with axial $g$ factors, i.e. with $\mathrm{g}_{\mathrm{x}}=\mathrm{g}_{\mathrm{y}}$.

\section{LPMO catalyzed oxidation of PASC}

PASC microcrystalline cellulose substrate was prepared by treating avicel with phosphoric acid as described by Wood et al. (1988) ${ }^{47} .4$ grams of Avicel was dispersed into $100 \mathrm{~mL}$ of phosphoric acid $(86 \% \mathrm{w} / \mathrm{v})$ at $60{ }^{\circ} \mathrm{C}$ and magnetically stirred for an hour. $1900 \mathrm{~mL}$ Milli-Q water was slowly dripped into the solution under stirring condition. Then suspension was transferred to $4{ }^{\circ} \mathrm{C}$ for sedimentation. After sedimentation, the supernatant was removed and the suspension was washed four times with Milli-Q water. The solution was neutralized using $\mathrm{Na}_{2} \mathrm{CO}_{3}$.

TaLPMO9A catalyzed oxidation of PASC contains TaLPMO9A $(0.1 \mathrm{mM})$, ascorbate $(2 \mathrm{mM})$, and PASC $(0.4 \%, \mathrm{w} / \mathrm{v})^{48}$. Reaction was carried out in absence or presence of $\mathrm{H}_{2} \mathrm{O}_{2}$ 
$(0.1 \mathrm{mM} / 2 \mathrm{mM})$ in $20 \mathrm{mM}$ phosphate buffer $(\mathrm{pH} 6.0)$ at $25^{\circ} \mathrm{C}$ for $30 \mathrm{~min}$. Reaction was stopped using $0.1 \mathrm{M} \mathrm{NaOH}$ and then samples were centrifuged. Supernatant was separated, filtered $(0.22 \mu \mathrm{M})$ and analyzed for oligosaccharide by HPAEC. HPAEC was run on an ICS5000 system equipped with a PAD detector (Dionex, Sunnyvale, CA, USA) with a CarboPac PA1 column (2 X $50 \mathrm{~mm}$ guard column followed by a 2 X $250 \mathrm{~mm}$ analytical column).

\section{High-Performance Anion Exchange Chromatography (HPAEC)}

HPAEC analysis of the released oligosaccharides from the cellulosic substrate was performed on an ICS-5000+ system, equipped with a PAD detector (Thermo Scientific), with a CarboPac PA1 column (two 2 x $50 \mathrm{~mm}$ guard columns followed by a 2 × $250 \mathrm{~mm}$ analytical column), and operated at $0.25 \mathrm{ml} \mathrm{min}^{-1}$ and $30^{\circ} \mathrm{C}$. The chromatographic separation of aldonic acids was carried out as described by Westering et al. (1913) ${ }^{49}$. For the elution the following gradient was applied (Eluent A, 0.1M NaOH; Eluent B, $1 \mathrm{M} \mathrm{NaOAc}$ in $0.1 \mathrm{M} \mathrm{NaOH}$ ): $100 \%$ A:0\% B to $90 \% \mathrm{~A}: 10 \% \mathrm{~B}(10 \mathrm{~min})$, then to $70 \% \mathrm{~A}: 30 \% \mathrm{~B}$ (25 min) and lastly 0\% A:100\% B (30 min). For reconditioning of the column 100\% A:0\% B was applied for 15 min (39-54 min). The cellooligosaccharide peaks were annotated according to elution profiles of commercially available non-oxidized oligosaccharides (DP2-DP6). The C4-oxidized glucose (C4-keto aldose) peaks appeared after $25 \mathrm{~min}$.

\section{LPMO Stopped-flow kinetics}

Fast kinetic studies were performed using a $\pi^{*-180}$ stopped-flow spectrometer (Applied Photophysics, Leatherhead, UK) with an observation cell of the light path length of $2 \mathrm{~mm}$.

Calibrations of the zero time-point and dead-time (the time from the zero time to the first good data point) were done by mixing 2,6-Dichlorophenolindophenol (DCIP) with different concentrations of excess L-ascorbic acid (AA) (under pseudo-first order reaction conditions) at a 1:1 ratio (v:v). Absorption was measured at $524 \mathrm{~nm}$, data acquisition and 
analysis were done using the Pro-Data software (Applied Photophysics). The zero time-point and dead-time may change with AA concentration, and all analysis were based on kinetic traces that were adjusted to actual time points of mixing determined by DCIP-AA experiments at different AA concentrations. The experimental data were fitted to single exponential equations. All the initial data points were omitted from the traces until satisfactory fits to single exponentials were obtained, and a common point of intersection was achieved for a range of AA concentrations. The point of intersection of the fitted curves is the true zero time for the mixing.

Kinetic studies of fast reactions between LPMO, AA and $\mathrm{H}_{2} \mathrm{O}_{2}$ were performed and the reaction species were followed at $420 \mathrm{~nm}$ with time scales from 60 to $1000 \mathrm{~s}$ at $20{ }^{\circ} \mathrm{C}$. The first stopped-flow syringe contained reduced TaLPMO9A, and second syringe contained varying concentrations of $\mathrm{H}_{2} \mathrm{O}_{2}(0.05-3.0 \mathrm{mM})$. The fit to the data was made between 5-1000 s thereby removing the rising part of the transients from the fit. The fit was made using the standard double exponential equation (Eq. 2).

$$
A_{420}=\mathrm{a} 1 \cdot \exp \left(-k_{1} \cdot \mathrm{x}\right)+\mathrm{a} 2 \cdot \exp \left(-k_{2} \cdot \mathrm{x}\right)+\mathrm{c} \quad \text { Eq. } 2
$$

where $k_{1}$, and $k_{2}$ are the observed rate constants for the fast and slow phases respectively, a1 and $\mathrm{a} 2$ are the relative amplitude values for the two phases, and c is an offset value to account for a non-zero baseline. Baseline tend to fluctuate during multiple shots. Therefore, baselines were normalized. All experiments were done in duplicates for each reactant concentration. After rapid mixing, absorbance changes were monitored at a $420 \mathrm{~nm}$ and appropriate controls were recorded in all cases to exclude the possibility of artifacts. 


\section{References}

1 Phillips, C. M., Beeson, W. T., Cate, J. H. \& Marletta, M. A. Cellobiose dehydrogenase and a copper-dependent polysaccharide monooxygenase potentiate cellulose degradation by Neurospora crassa. ACS Chem. Biol. 6, 1399-1406 (2011).

2 Sabbadin, F. et al. An ancient family of lytic polysaccharide monooxygenases with roles in arthropod development and biomass digestion. Nat. Commun. 9, 756 (2018).

3 Levasseur, A., Drula, E., Lombard, V., Coutinho, P. M. \& Henrissat, B. Expansion of the enzymatic repertoire of the CAZy database to integrate auxiliary redox enzymes. Biotechnol. Biofuels 6, 41 (2013).

4 Hemsworth, G. R., Henrissat, B., Davies, G. J. \& Walton, P. H. Discovery and characterization of a new family of lytic polysaccharide monooxygenases. Nat. Chem. Biol. 10, 122-126 (2014).

5 Quinlan, R. J. et al. Insights into the oxidative degradation of cellulose by a copper metalloenzyme that exploits biomass components. Proc. Natl. Acad. Sci. U. S. A. 108, 15079-15084 (2011).

6 Book, A. J. et al. Evolution of substrate specificity in bacterial AA10 lytic polysaccharide monooxygenases. Biotechnol. Biofuels 7, 109 (2014).

7 Kim, S., Stahlberg, J., Sandgren, M., Paton, R. S. \& Beckham, G. T. Quantum mechanical calculations suggest that lytic polysaccharide monooxygenases use a copper-oxyl, oxygen-rebound mechanism. Proc. Natl. Acad. Sci. U. S. A. 111, 149-154 (2014).

8 Beeson, W. T., Vu, V. V., Span, E. A., Phillips, C. M. \& Marletta, M. A. Cellulose degradation by polysaccharide monooxygenases. Annu. Rev. Biochem. 84, 923-946 (2015). 
9 O'Dell, W. B., Agarwal, P. K. \& Meilleur, F. Oxygen activation at the active site of a fungal lytic polysaccharide monooxygenase. Angew. Chem. Int. Edit. 56, 767-770 (2017).

10 Johansen, K. S. Lytic polysaccharide monooxygenases: the microbial power tool for lignocellulose degradation. Trends. Plant Sci. 21, 926-936 (2016).

11 Frommhagen, M. et al. Boosting LPMO-driven lignocellulose degradation by polyphenol oxidase-activated lignin building blocks. Biotechnol. Biofuels 10, 121 (2017).

12 Cannella, D. et al. Light-driven oxidation of polysaccharides by photosynthetic pigments and a metalloenzyme. Nat. Commun. 7, (2016).

13 Frommhagen, M. et al. Lytic polysaccharide monooxygenases from Myceliophthora thermophila $\mathrm{C} 1$ differ in substrate preference and reducing agent specificity. Biotechnol. Biofuels 9, 186 (2016).

14 Kracher, D. et al. Extracellular electron transfer systems fuel cellulose oxidative degradation. Science 352, 1098-1101 (2016).

15 Bissaro, B. et al. Oxidative cleavage of polysaccharides by monocopper enzymes depends on $\mathrm{H}_{2} \mathrm{O}_{2}$. Nat. Chem. Biol. 13, 1123-1128 (2017).

16 Vaaje-Kolstad, G., Forsberg, Z., Loose, J. S., Bissaro, B. \& Eijsink, V. G. Structural diversity of lytic polysaccharide monooxygenases. Curr. Opin. Struct. Biol. 44, 67-76 (2017).

17 Hangasky, J. A., Iavarone, A. T. \& Marletta, M. A. Reactivity of $\mathrm{O}_{2}$ versus $\mathrm{H}_{2} \mathrm{O}_{2}$ with polysaccharide monooxygenases. Proc. Natl. Acad. Sci. U. S. A. 115, 4915-4920 (2018).

18 Kuusk, S. et al. Kinetics of $\mathrm{H}_{2} \mathrm{O}_{2}$-driven degradation of chitin by a bacterial lytic polysaccharide monooxygenase. J. Biol. Chem. 293, 12284 (2018). 
19 Kjaergaard, C. H. et al. Spectroscopic and computational insight into the activation of $\mathrm{O}_{2}$ by the mononuclear $\mathrm{Cu}$ center in polysaccharide monooxygenases. Proc. Natl. Acad. Sci. U. S. A. 111, 8797-8802 (2014).

20 Bacik, J. P. et al. Neutron and high-resolution room-temperature X-ray data collection from crystallized lytic polysaccharide monooxygenase. Acta. Crystallogr. F. Struct. Biol. Commun. 71, 1448-1452 (2015).

$21 \mathrm{Wu}, \mathrm{M}$. et al. Crystal structure and computational characterization of the lytic polysaccharide monooxygenase GH61D from the Basidiomycota fungus Phanerochaete chrysosporium. J. Biol. Chem. 288, 12828-12839 (2013).

22 Frandsen, K. E. et al. The molecular basis of polysaccharide cleavage by lytic polysaccharide monooxygenases. Nat. Chem. Biol. 12, 298-303 (2016).

23 Bertini, L. et al. Catalytic mechanism of fungal lytic polysaccharide monooxygenases investigated by first-principles calculations. Inorg. Chem. 57, 86-97 (2018).

24 Wang, B. J. et al. QM/MM studies into the $\mathrm{H}_{2} \mathrm{O}_{2}$-dependent activity of lytic polysaccharide monooxygenases: evidence for the formation of a caged hydroxyl radical intermediate. ACS Catal. 8, 1346-1351 (2018).

25 Hedegard, E. D. \& Ryde, U. Targeting the reactive intermediate in polysaccharide monooxygenases. J. Biol. Inorg. Chem. 22, 1029-1037 (2017).

26 Hedegard, E. D. \& Ryde, U. Molecular mechanism of lytic polysaccharide monooxygenases. Chem. Sci. 9, 3866-3880 (2018).

27 Walton, P. H. \& Davies, G. J. On the catalytic mechanisms of lytic polysaccharide monooxygenases. Curr. Opin. Chem. Biol. 31, 195-207 (2016).

28 McDonald, M. R., Fredericks, F. C. \& Margerum, D. W. Characterization of copper(III)-tetrapeptide complexes with histidine as the third residue. Inorg. Chem. 36, 3119-3124 (1997). 
29 Mcdonald, M. R., Scheper, W. M., Lee, H. D. \& Margerum, D. W. Copper(III) complexes of tripeptides with histidine and histamine as the 3rd residue. Inorg. Chem. 34, 229-237 (1995).

30 Burke, S. K., Xu, Y. \& Margerum, D. W. Cu(II)Gly2HisGly oxidation by $\mathrm{H}_{2} \mathrm{O}_{2}$ /ascorbic acid to the CuIII complex and its subsequent decay to alkene peptides. Inorg. Chem. 42, 5807-5817 (2003).

31 Tepper, A. W. et al. Identification of a radical intermediate in the enzymatic reduction of oxygen by a small laccase. J. Am. Chem. Soc. 131, 11680-11682 (2009).

32 Hangasky, J. A., Detomasi, T. C. \& Marletta, M. A. Glycosidic bond hydroxylation by polysaccharide monooxygenases. Trends in Chemistry (2019).

33 Tiwari, M. K. et al. Early events in copper-ion catalyzed oxidation of alpha-synuclein. Free Radic. Biol. Med. 121, 38-50 (2018).

34 Breslmayr, E. et al. A fast and sensitive activity assay for lytic polysaccharide monooxygenase. Biotechnol. Biofuels 11, 79 (2018).

35 Simmons, T. J. et al. Structural and electronic determinants of lytic polysaccharide monooxygenase reactivity on polysaccharide substrates. Nat. Commun. 8, 1064 (2017).

36 Skalova, T. et al. The structure of the small laccase from Streptomyces coelicolor reveals a link between laccases and nitrite reductases. J. Mol. Biol. 385, 1165-1178 (2009).

37 Gupta, A. et al. Involvement of Tyr108 in the enzyme mechanism of the small laccase from Streptomyces coelicolor. J. Am. Chem. Soc. 134, 18213-18216 (2012).

38 Lo Leggio, L., Welner, D. \& De Maria, L. A structural overview of GH61 proteins fungal cellulose degrading polysaccharide monooxygenases. Comput. Struct. Biotechnol. J. 2, (2012). 
39 Li, X., Beeson, W. T. t., Phillips, C. M., Marletta, M. A. \& Cate, J. H. Structural basis for substrate targeting and catalysis by fungal polysaccharide monooxygenases. Structure 20, 1051-1061 (2012).

40 Frommhagen, M., Westphal, A. H., van Berkel, W. J. H. \& Kabel, M. A. Distinct substrate specificities and electron-donating systems of fungal lytic polysaccharide monooxygenases. Front. Microbiol. 9, 1080 (2018).

41 Nekiunaite, L., Arntzen, M. O., Svensson, B., Vaaje-Kolstad, G. \& Abou Hachem, M. Lytic polysaccharide monooxygenases and other oxidative enzymes are abundantly secreted by Aspergillus nidulans grown on different starches. Biotechnol. Biofuels $\mathbf{9}$, 187 (2016).

42 Caldararu, O., Oksanen, E., Ryde, U. \& Hedegard, E. D. Mechanism of hydrogen peroxide formation by lytic polysaccharide monooxygenase. Chem. Sci. 10, 576-586 (2019).

43 Isaksen, T. et al. A C4-oxidizing lytic polysaccharide monooxygenase cleaving both cellulose and cello-oligosaccharides. J. Biol. Chem. 289, 2632-2642 (2014).

44 Kittl, R., Kracher, D., Burgstaller, D., Haltrich, D. \& Ludwig, R. Production of four Neurospora crassa lytic polysaccharide monooxygenases in Pichia pastoris monitored by a fluorimetric assay. Biotechnol. Biofuels 5, 79 (2012).

45 Beers, R. F., Jr. \& Sizer, I. W. A spectrophotometric method for measuring the breakdown of hydrogen peroxide by catalase. J. Biol. Chem. 195, 133-140 (1952).

46 Piligkos, S., Laursen, I., Morgenstjerne, A. \& Weihe, H. Sign and magnitude of spin Hamiltonian parameters for $\mathrm{Mn}^{2+}$ impurities in calcite. A multi- and low-frequency EPR study. Mol. Phys. 105, 2025-2030 (2007).

47 Wood, T. M. Preparation of crystalline, amorphous, and dyed cellulase substrates. Method. Enzymol. 160, 19-25 (1988). 
48 Mollers, K. B. et al. On the formation and role of reactive oxygen species in lightdriven LPMO oxidation of phosphoric acid swollen cellulose. Carbohydr. Res. $\mathbf{4 4 8 ,}$ 182-186 (2017).

49 Westereng, B. et al. Efficient separation of oxidized cello-oligosaccharides generated by cellulose degrading lytic polysaccharide monooxygenases. J. Chromatogr. A 1271, 144-152 (2013). 


\section{Acknowledgments}

This work is supported by the Novo Nordisk Foundation project "Harnessing the Energy of the Sun for Biomass Conversion" (NNF16OC0021832). We also acknowledge the donation of enzymes from Novozymes A/S. All the data relevant to this study are included in the main paper or the supplementary materials.

\section{Authors contributions}

M. J. B. and R. K. S. designed, performed and analyzed most of the experiments. R. S. performed the stopped-flow kinetics experiments. B. M. and H. W. helped to perform and analyze the PASC oxidation and EPR spectroscopy data, respectively. B.v.O helped to analyse the stopped-flow data. M. J. B. and R. K. S. wrote the manuscript with support from C. F. and D. A. R. All authors participated in critical analysis of the data and finalizing the manuscript.

\section{Competing financial interests}

The authors declare no competing financial interests. 